\title{
Evaluación del componente bucal. Proyecto "Escuelas Saludables" de la Fundación las Golondrinas, MEDELlín, 2006-2011
}

\author{
Martha Yojana Gómez-Arias, odontóloga ${ }_{1}$, Liliana Yamile Franco-Londoño, odontóloga \\ José Miguel Gaviria-Torres, odontólogo ${ }_{1}$, Lina Marcela Baena-Muñoz, odontóloga, \\ Juan Fernando Ortiz-Restrepo, odontólogo ${ }_{1}$, Julieth Paulina Zapata-Bedoya, odontóloga ${ }_{1}$, \\ Tomás Bernal-Álvarez, Esp., MSc ${ }_{1}$, Andrés A. Agudelo-Suárez ${ }^{*}$ Esp., Ph.D. ${ }_{1}$
}

${ }_{1}$ Facultad de Odontología, Universidad de Antioquia, Colombia

Recibido: 25 de febrero del 2013. Aprobado: 2 de abril del 2013.

*Autor de correspondencia: Andrés Agudelo Suárez, Facultad de Odontología, Universidad de Antioquia, Medellín, Colombia, (57) 4 219 6772 , Calle 64 n. ${ }^{\circ} 52-59$, correo electrónico: oleduga@gmail.com

Cómo citar este artículo: Gómez-Arias MY, Franco-Londoño LY, Gaviria-Torres JM, Baena-Muñoz LM, Ortiz Restrepo JF, Zapata-Bedoya JP, Bernal-Álvarez T, AgudeloSuarez AA. Evaluación del componente bucal. Proyecto "Escuelas Saludables" de la Fundación las Golondrinas, Medellín, 2006-2011. Revista Nacional de Odontología. 2013; 9(16): 15-26.

Resumen. Introducción: se pretende evaluar una estrategia de escuela saludable en su componente bucal a través de indicadores de higiene bucal, salud bucal y conocimientos y prácticas de diversos actores (escolares y padres o adultos significativos). Métodos: estudio evaluativo a través de elementos de un estudio cuantitativo y descriptivo con datos de dos momentos del tiempo (2006 y 2011). Se revisaron historias clínicas y se realizaron exámenes clínicos para indicadores de higiene bucal (IHO) y salud bucal (COP-D, ceo-d). Encuesta a padres o adultos significativos, y a escolares, sobre conocimientos y prácticas en salud bucal. Resultados: existe mejoría en los conocimientos y prácticas de los escolares partícipes del desarrollo del proyecto, haciéndose visible para los adultos que notan un cambio positivo en la salud bucal de los niños. Se presentó un cambio positivo en la higiene bucal del $56 \%$ de las mujeres, del $54 \%$ de los niños y niñas menores de 11 años y del 53\% de los estudiantes de secundaria. Se encontró para el 2011 que el $60 \%$ estaba sano en el momento del examen y un $44 \%$ no tenía experiencia de caries. Conclusiones: los indicadores observados permitieron evaluar positivamente los resultados del proyecto, y se invita al fortalecimiento de estrategias comunitarias desde la educación para la salud en los planes educativos institucionales.

Palabras clave: educación en salud dental, educación para la salud, higiene bucal, promoción de la salud, salud bucal.

\section{Evaluation of the Oral Component. "Healthy Schools" ("Escuelas Saludables") Project from the "Fundación las Golondrinas", Medellín, 2006-2011}

Abstract. Introduction: We aim to assess a health education strategy for
schools, regarding the oral component, through oral hygiene and oral
health indexes, using the knowledge and practices for oral health of
school kids, parents and/or significant adults. Methods: We conducted
an evaluation using elements from a quantitative and descriptive study
in two periods, 2006 and 2011 . We carried out a review of dental records
and clinical examinations using oral hygiene (IHOs) and oral health
(DMFT and deft) indexes. A survey was conducted in school kids, parents
and/or significant adults about knowledge and practices relevant to oral
health. Results: We found an improvement of the knowledge and prac-
tices relating to oral health in the participants of the study. Parents and
adults noticed a positive change in the oral health of children. A positive
change in the oral hygiene was found in $56 \%$ of females, $54 \%$ of children
under 11 and $53 \%$ of the high school students. $60 \%$ of the children were
healthy relative to cavities at the time of examination and $44 \%$ had no
caries experience. Conclusions: The observed indicators allowed us to
positively evaluate the results of the project and invited to strengthen-
ing community strategies for health education within institutional plans.

Keywords: dental health education, health education, oral hygiene, health promotion, oral health.
Avaliação do componente bucal. Projeto "Escolas Saudáveis" da Fundação Las Golondrinas, Medellín, 2006-2011

Resumo. Introdução: pretende-se avaliar uma estratégia de escola saudável em seu componente bucal por meio de indicadores de higiene bucal, saúde bucal e conhecimentos e práticas de diversos autores (escolares e pais ou adultos significativos). Métodos: estudo avaliativo mediante elementos de um estudo quantitativo e descritivo com dados de dois momentos: 2006 e 2011. Revisaram-se histórias clínicas e realizaram-se exames clínicos para indicadores de higiene bucal (IHOs) e saúde bucal (COP-D, ceo-d). Enquete a pais ou adultos significativos e a escolares sobre conhecimentos e práticas em saúde bucal. Resultados: existe uma melhoria nos conhecimentos e práticas dos escolares participantes do desenvolvimento do projeto, fazendo-se visível para os adultos que notam uma mudança positiva na saúde bucal das crianças. Apresentou-se uma mudança positiva na higiene bucal de $56 \%$ das mulheres, de $54 \%$ das crianças menores de 11 anos e de 53\% dos estudantes do ensino fundamental (a partir da sexta série). Constatou-se para 2011 que $60 \%$ estavam saudáveis no momento do exame e $44 \%$ não tinham experiência de cáries. Conclusões: os indicadores observados permitiram avaliar positivamente os resultados do projeto, e convida ao fortalecimento de estratégias comunitárias a partir da educação para a saúde nos planos educativos institucionais.

Palavras-chave: educação em saúde dental, educação para a saúde, higiene bucal, promoção da saúde, saúde bucal. 


\section{Introducción}

A través del tiempo se ha demostrado que la educación para la salud es una de las estrategias más efectivas para promover la salud y prevenir la enfermedad, al buscar que los individuos de una comunidad adopten hábitos saludables que les permita mejorar su calidad de vida $[1,2]$. Específicamente en salud bucal se pretende fomentar los conocimientos, actitudes y prácticas que permitan a la sociedad tomar conciencia de todas aquellas alteraciones que se manifiestan a causa de una inadecuada higiene bucal $[3,4]$.

La Fundación las Golondrinas está ubicada en las comunas 8 y 9 , zona centro oriental de la ciudad de Medellín. Fue fundada hace 31 años, y es considerada una organización no gubernamental (ONG) que trabaja con población desplazada y marginada. En la institución se prestan los servicios de educación, salud y desarrollo comunitario [5], para los cuales han realizado diferentes convenios con la Secretaría de Educación, Bienestar y Desarrollo Social, Instituto Colombiano de Bienestar Familiar (ICBF), el SENA, la Universidad de Antioquia, entre otros.

Desde el 2006, esta fundación, en convenio con la Facultad de Odontología de la Universidad de Antioquia [6], viene desarrollando actividades de promoción de la salud y prevención de la enfermedad, con el fin de mejorar los conocimientos, actitudes y prácticas en salud, y particularmente en higiene bucal, así como acciones de curación y rehabilitación en salud bucal. En estas actividades se ha contado con la participación de la comunidad educativa (profesores, directivas, estudiantes y padres y adultos significativos). Estas acciones han permeado en otros servicios y áreas del conocimiento, como por ejemplo: nutrición, medicina, educación física, psicología, trabajo social [5].

Como parte importante de la estrategia de educación para la salud, en esta fundación se pretende realizar una evaluación de la estructura, el proceso y los resultados de las diferentes estrategias empleadas a lo largo de estos años. En una primera parte, se evaluarán los resultados de las acciones llevadas a cabo y que tienen relación con educación específica en salud bucal, conocimientos y prácticas de la comunidad educativa, con el fin de emprender nuevas acciones y estrategias que ayuden a continuar o reorientar el convenio entre las instituciones.

La literatura ha reportado el impacto positivo que se puede lograr en una comunidad por medio de las intervenciones educativas [7-11], haciendo evidente los puntos clave en los que se debe intervenir como actores de la salud, y las debilidades o puntos en los cuales se requiere también una participación política y social. Se ha encontrado que el nivel educativo, el nivel socioeconómico y la conducta cultural son factores determinantes en la salud de las comunidades [12]. De esta manera se puede afirmar que implementando proyectos y programas dirigidos a mejorar estos determinantes, se logran mejorar las condiciones de salud de una comunidad, específicamente en lo que tiene que ver con salud bucal [13].

El objetivo de este estudio es, por consiguiente, evaluar los resultados de la propuesta de intervención en salud bucal, realizada en el marco del proyecto La Educación en Salud, una opción curricular en el Proyecto de Escuela Saludable para la Fundación las Golondrinas, Medellín, Antioquia, teniendo en cuenta: 1) los conocimientos y prácticas en salud bucal en escolares y padres de familia o adultos significativos; y 2) situación de higiene bucal y salud bucal de la población escolar.

\section{Métodos}

Se trata de un estudio evaluativo, el cual utiliza elementos de un estudio cuantitativo y descriptivo con datos de dos momentos del tiempo (2006 y 2011). La población de estudio la constituyeron los escolares de la Fundación las Golondrinas, ubicada en la comuna 8 de la ciudad de Medellín, que iniciaron el programa en el 2006. Luego de consultar las historias clínicas odontológicas de la población escolar, se logró recopilar información de 59 niños y niñas. Posteriormente, se ubicaron los niños que continuaban en la institución y se logró evaluar a 48 niños. También se incluyeron 24 padres y adultos significativos o acudientes de niños y niñas que voluntariamente aceptaron participar en esta investigación.

El trabajo de campo lo llevó a cabo el grupo investigador, y se aplicaron varios instrumentos de recolección de la información: 1) recopilación de información secundaria de indicadores para el 2006: a) índice de higiene oral simplificado de Green y Vermillon (IHO) [14]; este es un indicador que mide la presencia de placa blanda en dientes deciduos y permanentes; se registra el promedio y va en un rango de 0 a 3,0 (a más promedio más presencia de placa); b) índices ceo-d y COP-D: indica la experiencia y la prevalencia de caries dental tanto para dientes deciduos como permanentes 
[15, 16]; 2) examen clínico: se tomaron los índices de IHO y COP-D para el 2011 ; 3) instrumento de conocimientos y prácticas en salud bucal: el grupo investigador diseñó y aplicó dos instrumentos para padres o adultos significativos o acudientes y para los escolares (disponible si se solicita a los autores). El instrumento para padres y cuidadores contiene 37 preguntas sobre datos sociodemográficos, conocimientos sobre salud bucal, creencias, prácticas rutinarias en higiene bucal y algunas prácticas con respecto a la educación para la salud bucal de los niños y niñas, al igual que opinión sobre el programa realizado en la escuela. De otra parte, el instrumento para la población escolar contiene 28 preguntas con información similar a la de los padres; es importante señalar que muchas de las preguntas son iguales, lo cual facilita su comparación.

Se realizó un análisis descriptivo para las variables relacionadas con salud bucal. En el caso del índice IHO, se calculó el promedio y la mediana para el 2006 y el 2011 total, y según sexo, edad y nivel educativo. Se calculó en porcentaje el tipo de cambio en las condiciones de higiene bucal (positivo, negativo o no cambio). Para el caso del ceo-d inicial (2006) y el cop-D final se calcularon promedios con sus intervalos de confianza al $95 \%\left(\mathrm{IC}_{95 \%}\right)$, total y según edad, nivel educativo y sexo. En todos los casos se hicieron pruebas Chi cuadrado ( $t$ de student para muestras independientes) con el fin de observar diferencias significativas en las diferentes variables. Se calculó el porcentaje de niños sin caries en el momento del examen, y sin experiencia de caries (nunca han tenido caries, no tienen dientes obturados o perdidos por caries) según variables, y pruebas Chi cuadrado de diferencia de proporciones. Para el caso de conocimientos y prácticas se realizó un análisis descriptivo para las variables de las encuestas según actor social (niño(a), padre o adulto significativo).

Según la normativa internacional (Declaración de Helsinki) [17] y nacional (Resolución 008430 de 1993 del Ministerio de Salud) [18] sobre investigación en seres humanos, se considera una investigación sin riesgo, puesto que no se interviene en las características físicas, psicológicas o sociales de los pacientes seleccionados en el estudio. Se obtuvo consentimiento de las directivas de la institución y de los padres o adultos significativos y de los escolares para participar en el estudio, y se garantizó protección de la identidad de la población entrevistada, confidencialidad y veracidad de la información. Este estudio fue aprobado como un ejercicio académico de seis de los coautores en el pregrado de Odontología de la Universidad de Antioquia, Medellín.

\section{Resultados}

\section{Características sociodemográficas \\ de la población escolar y de los padres o adultos significativos}

La evaluación de indicadores de higiene bucal en el periodo inicial y final fue realizada a 48 escolares (mujeres: $\mathrm{n}=27 ; 56,3 \%$ ). El $54 \%$ corresponde a menores de 11 años $(n=26)$. El 60,4\% $(n=29)$ se encuentra cursando primaria (tercero, cuarto y quinto) y el $39,6 \%$ $(\mathrm{n}=19)$, en secundaria (sexto, séptimo y octavo). Participaron 24 padres y cuidadores (mujeres: $\mathrm{n}=18 ; 75 \%$ ). El 50\% ( $\mathrm{n}=12)$ es menor de 35 años. El 54\% $(\mathrm{n}=13)$ tiene estudios primarios o menores, y sólo el 4,2 $(\mathrm{n}=1)$ cuenta con estudios tecnológicos (educación superior). El $46 \%(\mathrm{n}=11)$ pertenece al régimen subsidiado, el $42 \%(\mathrm{n}=10)$, al régimen contributivo, y el $12 \%(\mathrm{n}=3)$ no cuenta con afiliación al sGsss.

\section{Situación de salud bucal e higiene bucal en la población escolar}

En la tabla 1 se muestra el promedio, la mediana y el tipo de cambio en los indicadores de higiene oral. En términos generales, se observó un ligero descenso en el

Tabla 1. Indicador de higiene bucal en los dos periodos evaluados, según diferentes características sociodemográficas. Fundación las Golondrinas, Medellín

\begin{tabular}{|c|c|c|c|c|c|c|c|c|c|}
\hline \multirow{3}{*}{\multicolumn{2}{|c|}{ Variables }} & \multirow{3}{*}{ Muestra } & \multicolumn{7}{|c|}{ IHO } \\
\hline & & & \multicolumn{2}{|c|}{ Promedio } & \multicolumn{2}{|c|}{ Mediana } & \multicolumn{3}{|c|}{ Cambio (porcentaje) } \\
\hline & & & Inicial & Final & Inicial & Final & Positivo & Negativo & No cambio \\
\hline \multirow{2}{*}{ Sexo } & Hombres & 21 & 1,48 & 1,52 & 1,30 & 1,50 & 47,6 & 47,6 & 4,8 \\
\hline & Mujeres & 27 & 1,46 & 1,30 & 1,33 & 1,40 & 55,6 & 40,7 & 3,7 \\
\hline \multirow{2}{*}{ Edad } & $7-10$ & 26 & 1,51 & 1,49 & 1,23 & 1,50 & 53,8 & 42,3 & 3,8 \\
\hline & $>=11$ & 22 & 1,43 & 1,28 & 1,42 & 1,40 & 50,0 & 45,5 & 4,5 \\
\hline
\end{tabular}




\begin{tabular}{|c|c|c|c|c|c|c|c|c|c|}
\hline \multirow{3}{*}{\multicolumn{2}{|c|}{ Variables }} & \multirow{4}{*}{$\begin{array}{c}\text { Muestra } \\
29\end{array}$} & \multicolumn{7}{|c|}{ IHO } \\
\hline & & & \multicolumn{2}{|c|}{ Promedio } & \multicolumn{2}{|c|}{ Mediana } & \multicolumn{3}{|c|}{ Cambio (porcentaje) } \\
\hline & & & Inicial & Final & Inicial & Final & Positivo & Negativo & No cambio \\
\hline \multirow{3}{*}{ Grado escolar } & Primaria & & 1,44 & 1,47 & 1,16 & 1,50 & 51,7 & 48,3 & 0,0 \\
\hline & Secundaria & 19 & 1,52 & 1,29 & 1,60 & 1,50 & 52,6 & 36,8 & 10,5 \\
\hline & Total & 48 & 1,47 & 1,39 & 1,32 & 1,50 & 52,1 & 43,8 & 4,2 \\
\hline
\end{tabular}

Fuente: elaboración propia

promedio entre los dos periodos evaluados, el cual fue más evidente en las mujeres, en la población escolar $\geq 11$ años y de secundaria, aunque no se presentaron diferencias significativas. Esto representó un cambio positivo en la higiene bucal del $56 \%$ de las mujeres, del $54 \%$ de los niños y niñas menores de 11 años, y del 53\% de los estudiantes de secundaria.

Según los indicadores de caries dental para niños y niñas (tabla 2), el promedio de ceo-d al inicio del programa fue de 2,4 ( $\left.\mathrm{IC}_{95 \%}, 1,4-2,3\right)$; este indicador fue mayor en niños y en aquellos que tienen menor nivel educativo y se encontraron diferencias estadísticamente significativas. El COP-D actual observado fue de $1,3\left(\mathrm{IC}_{95 \%} 0,9-1,7\right)$, ligeramente mayor en niñas pero sin diferencias estadísticamente significativas, y ligeramente mayor en la población de secundaria y con diferencias significativas. Al inicio del programa, un $42 \%$ de la población escolar no tenía experiencia de caries (sanos) en dentición decidua; en el periodo actual se encontró que el 60\% estaba sano en el momento del examen, y el 44\% no tenía experiencia de caries (tabla 2).

\section{Conocimientos y prácticas en salud bucal en los escolares y en los padres y adultos significativos}

Tras analizar los conocimientos en salud bucal, tanto en escolares como en sus padres o adultos significativos (tabla 3), se encontró que, con respecto a la pregunta de "quién es el odontólogo", más de la mitad de la población escolar encuestada piensa que es la persona que enseña cómo cuidarse los dientes, mientras que más de dos tercios de los padres piensan que es la

Tabla 2. Indicadores de caries dental (ceo-d y COP-D) e indicadores de salud bucal ${ }^{\star}$ en los dos periodos evaluados, según diferentes características sociodemográficas. Fundación las Golondrinas, Medellín

\begin{tabular}{|c|c|c|c|c|c|c|c|c|c|}
\hline \multirow{2}{*}{\multicolumn{2}{|c|}{ Variables }} & \multicolumn{2}{|c|}{ Promedio $\left(\mathrm{IC}_{95 \%}\right)$} & \multicolumn{3}{|c|}{ Sin caries $(C / c=0)$} & \multicolumn{3}{|c|}{$\begin{array}{l}\text { Sin experiencia de caries } \\
\quad(\text { COP-D } / \text { ceo-d }=0)\end{array}$} \\
\hline & & ceo/COP & $p$ & $\mathrm{n}$ & $\%$ & $p$ & $\mathrm{n}$ & $\%$ & $p$ \\
\hline \multicolumn{10}{|c|}{ ceo-d $(n=48)$ inicial } \\
\hline \multirow{2}{*}{ Sexo } & Niños & $3,29(1,49-5,08)$ & \multirow{2}{*}{0,016} & 7 & 33,3 & \multirow{2}{*}{0,136} & 7 & 33,3 & \multirow{2}{*}{0,461} \\
\hline & Niñas & $1,67(0,72-2,61)$ & & 16 & 59,3 & & 13 & 48,1 & \\
\hline \multirow{2}{*}{ Edad } & $7-10$ & $2,92(1,61-4,24)$ & \multirow{2}{*}{0,345} & 9 & 34,6 & \multirow{2}{*}{0,086} & 7 & 26,9 & \multirow{2}{*}{0,05} \\
\hline & $\geq 11$ & $1,73(0,33-3,12)$ & & 14 & 63,6 & & 13 & 59,1 & \\
\hline \multirow{3}{*}{ Grado escolar } & Primaria & $3,10(1,70-4,51)$ & \multirow{2}{*}{0,008} & 10 & 34,5 & \multirow{2}{*}{0,045} & 9 & 31,0 & \multirow{2}{*}{0,122} \\
\hline & Secundaria & $1,26(0,31-2,21)$ & & 13 & 68,4 & & 11 & 57,9 & \\
\hline & Total & $2,38(1,44-2,31)$ & & 23 & 47,9 & & 20 & 41,7 & \\
\hline \multicolumn{10}{|c|}{ COP-D final $(n=48)$} \\
\hline \multirow{2}{*}{ Sexo } & Niños & $1,19(0,64-1,74)$ & \multirow{2}{*}{0,062} & 12 & 57,1 & \multirow{2}{*}{0,911} & 8 & 38,1 & \multirow{2}{*}{0,687} \\
\hline & Niñas & $1,37(0,68-2,07)$ & & 17 & 63,0 & & 13 & 48,1 & \\
\hline \multirow{2}{*}{ Edad } & $7-10$ & $1,38(0,76-2,31)$ & \multirow{2}{*}{0,983} & 15 & 57,7 & \multirow{2}{*}{0,902} & 10 & 38,5 & \multirow{2}{*}{0,609} \\
\hline & $\geq 11$ & $1,18(0,50-1,86)$ & & 14 & 63,6 & & 11 & 50,0 & \\
\hline \multirow{3}{*}{ Grado escolar } & Primaria & $1,17(0,71-1,64)$ & \multirow{2}{*}{0,022} & 16 & 55,2 & \multirow{2}{*}{0,538} & 12 & 41,4 & \multirow{2}{*}{0,911} \\
\hline & Secundaria & $1,47(0,55-2,40)$ & & 13 & 68,4 & & 9 & 47,4 & \\
\hline & Total & $1,29(0,85-1,74)$ & & 29 & 60,4 & & 21 & 43,8 & \\
\hline
\end{tabular}

*Se optó por incluir indicadores de salud positivos, que señalan el número y porcentaje de personas que se encontraban sanas para caries en el momento del examen y sin experiencia de caries dental (presente o actual)

Fuente: elaboración propia 
Tabla 3. Conocimientos en salud bucal en niños y padres y adultos significativos. Fundación las Golondrinas, Medellín

\begin{tabular}{|c|c|c|}
\hline Variables & Niños $(n=48)$ & Padres $(n=24)$ \\
\hline \multicolumn{3}{|l|}{ ¿Quién es el odontólogo? } \\
\hline La persona que hace dientes & 31,3 & 3,6 \\
\hline La persona que enseña cómo cuidarse los dientes & 52,1 & 10,7 \\
\hline La persona encargada de vigilar y controlar que las personas conserven una buena salud oral & 2,1 & 71,4 \\
\hline Ninguna de las anteriores & 14,6 & 0,0 \\
\hline \multicolumn{3}{|l|}{ ¿Qué es tener una boca sana? (respuestas afirmativas) } \\
\hline No tener dolor & 68,8 & 66,7 \\
\hline Tener caries & 2,1 & 8,3 \\
\hline Tener los dientes completos & 85,4 & 70,8 \\
\hline Tener mal aliento & 0,0 & 4,2 \\
\hline No tener placa bacteriana o cálculos & 31,3 & 58,3 \\
\hline Tener sangrado de encías & 2,1 & 0,0 \\
\hline \multicolumn{3}{|l|}{ ¿Hasta qué edad deben durar los dientes de una persona? } \\
\hline Años & 25,0 & 8,3 \\
\hline Toda la vida & 54,2 & 87,5 \\
\hline No sabe/No responde & 20,8 & 4,2 \\
\hline \multicolumn{3}{|l|}{ ¿Sabe qué es la higiene bucal? } \\
\hline Sí & 39,6 & 91,7 \\
\hline No & 60,4 & 8,3 \\
\hline \multicolumn{3}{|l|}{ ¿Qué es la placa bacteriana? (respuestas afirmativas) } \\
\hline Residuos de alimentos en los dientes & 60,4 & 79,2 \\
\hline Es una crema dental & 2,1 & 4,2 \\
\hline Microorganismos en los dientes & 23,0 & 75,0 \\
\hline Defectos del desarrollo de los dientes & 22,9 & 41,7 \\
\hline \multicolumn{3}{|l|}{ ¿Qué hace la placa bacteriana? (respuestas afirmativas) } \\
\hline Pone más blancos los dientes & 2,1 & 16,7 \\
\hline Produce la caries y el sangrado de la encía & 60,4 & 66,7 \\
\hline Hace más fuertes los dientes & 2,1 & 20,8 \\
\hline \multicolumn{3}{|l|}{ ¿Sabes qué es la caries dental? } \\
\hline Sí & 72,9 & 70,8 \\
\hline No & 27,1 & 29,2 \\
\hline \multicolumn{3}{|l|}{ ¿Por qué le da caries a los dientes? (respuestas afirmativas) } \\
\hline Mal cepillado o no cepillarse & 83,3 & 95,8 \\
\hline Consumo de dulces & 81,3 & 87,5 \\
\hline Usar la seda & 4,2 & 8,3 \\
\hline Buena alimentación & 8,3 & 12,5 \\
\hline \multicolumn{3}{|l|}{ ¿Qué daños puede producir la caries dental? (respuestas afirmativas) } \\
\hline Pone los dientes torcidos & 35,4 & 37,5 \\
\hline Mal aliento & 81,3 & 87,5 \\
\hline Dolor & 72,9 & 91,7 \\
\hline Pérdida de los dientes & 79,2 & 95,8 \\
\hline Pone los dientes fuertes & 4,2 & 4,2 \\
\hline Pone brillantes los dientes & 0,0 & 4,2 \\
\hline Fractura de los dientes & 75,0 & 50,0 \\
\hline \multicolumn{3}{|l|}{ ¿Cada cuánto se debe visitar al odontólogo? } \\
\hline Cada que siente dolor & 27,1 & 16,7 \\
\hline Cada 3 meses & 35,4 & 33,3 \\
\hline
\end{tabular}




\begin{tabular}{|c|c|c|}
\hline Variables & Niños $(n=48)$ & Padres $(n=24)$ \\
\hline Cada 6 meses & 18,8 & 29,2 \\
\hline Cada año & 4,2 & 8,3 \\
\hline Nunca lo visita & 8,3 & 0,0 \\
\hline No sabe/No responde & 6,3 & 12,5 \\
\hline \multicolumn{3}{|l|}{ ¿Cuándo se debe cambiar el cepillo de dientes? } \\
\hline Cuando está viejo & 25,0 & 0,0 \\
\hline Cuando está deshilachado & 27,1 & 20,8 \\
\hline Cuando no cepilla bien & 4,2 & 4,2 \\
\hline Cuando sale uno nuevo en el mercado & 2,1 & 0,0 \\
\hline Cuando se ve sucio & 10,4 & 4,2 \\
\hline Periódicamente & 29,2 & 70,8 \\
\hline Otra & 2,1 & 0,0 \\
\hline \multicolumn{3}{|l|}{ ¿Sabe qué es, para qué sirve el flúor? } \\
\hline Sí & 8,3 & 45,8 \\
\hline No & 91,7 & 54,2 \\
\hline
\end{tabular}

Fuente: elaboración propia

persona encargada de vigilar y controlar que las personas conserven una buena salud bucal. En cuanto a qué es "tener una boca sana", más del $60 \%$ de los escolares y adultos piensa que es no tener dolor; el $85 \%$ de los estudiantes encuestados y el $70 \%$ de los adultos afirman que es tener los dientes completos. En otra pregunta, más de la mitad de los escolares y cuatro quintos de los adultos afirman que los dientes de una persona deben durar toda la vida.

Tras preguntar que es la higiene bucal, el $60 \%$ de los escolares no sabe qué es, mientras la mayor parte de los adultos (91\%) refiere sí conocer qué es la higiene bucal. La mayoría de los escolares participantes y adultos afirma que la placa bacteriana es el residuo de alimentos en los dientes, y que esta produce la caries y el sangrado de la encía; también la mayoría de los niños y adultos afirma saber lo que es la caries dental; piensa que se produce por no cepillarse o hacerlo mal, y por comer dulces; afirma que puede producir mal aliento, dolor, pérdida de los dientes y fractura dental; finalmente, el 35\% de los estudiantes y el 33\% de los adultos afirman que cada tres meses se debe visitar al odontólogo.

Tabla 4. Prácticas en salud bucal en niños y padres y adultos significativos. Fundación las Golondrinas, Medellín

\begin{tabular}{|l|c|c|}
\hline \multicolumn{1}{|c|}{ Variables } & Niños (n= 48) & Padres $(\mathbf{n}=\mathbf{2 4})$ \\
\hline Uso de elementos para la higiene bucal (respuestas afirmativas) & & 100,0 \\
\hline Cepillo & 100,0 & 95,8 \\
\hline Crema dental & 97,1 & 83,3 \\
\hline Seda dental & 68,8 & 70,8 \\
\hline Enjuague bucal & 39,6 & 16,7 \\
\hline Palillos & 10,4 & 16,7 \\
\hline Bicarbonato & 14,6 & 0,0 \\
\hline Sal & 4,2 & \\
\hline ¿Cada cuánto se cepilla los dientes? (respuestas afirmativas) & & \\
\hline Al levantarse & 56,3 & \\
\hline Después del desayuno & 72,9 & \\
\hline Después del almuerzo & 68,8 & 58,3 \\
\hline Después de la comida & 64,6 & 91,7 \\
\hline Antes de acostarse & 60,4 & 75,0 \\
\hline & & 75,0 \\
\hline
\end{tabular}




Viene
\begin{tabular}{|l|r|r|}
\hline \multicolumn{1}{|c|}{ Variables } & Niños $(\mathbf{n}=\mathbf{4 8})$ & Padres $(\mathbf{n}=\mathbf{2 4})$ \\
\hline ¿Cuándo utiliza la seda dental? (respuestas afirmativas) & & \\
\hline No usa seda dental & 18,8 & 12,5 \\
\hline Cuando quedan restos de comida entre los dientes & 25,0 & 12,5 \\
\hline Cuando se acuerda & 6,3 & 0,0 \\
\hline Siempre que se cepilla, para completar la higiene oral & 27,1 & 66,7 \\
\hline De vez en cuando & 22,9 & 8,3 \\
\hline
\end{tabular}

Fuente: elaboración propia

Concretamente, en lo que tiene que ver con las prácticas de higiene bucal (tabla 4), se observó que la mayoría de la población utiliza los implementos de higiene bucal más comunes, como son el cepillo, la crema y la seda dental, con frecuencias que varían con diversidad entre adultos y escolares. Otros implementos que han sido tradicionalmente utilizados por la población como bicarbonato y palillos están siendo cada vez menos utilizados.

Con respecto a la opinión sobre el odontólogo y las actividades de la institución (tabla 5), tanto los escolares como los adultos encuestados en la fundación respondieron que ir al odontólogo era necesario e importante. El 92\% de los escolares y el 79\% de los padres respondieron que sí habían recibido información acerca del cui- dado de su salud bucal, y que esta información la han obtenido en su mayoría a través de charlas ofrecidas en el colegio. Finalmente, el $94 \%$ de los escolares y el $58 \%$ de los padres consideran que las charlas y actividades ofrecidas por la institución sobre temas de salud bucal han sido de su total agrado y se sienten muy satisfechos con estas.

\section{Prácticas de los padres y adultos significativos con respecto a la salud bucal de los escolares}

Tras el análisis de las respuestas de los padres en cuanto al cuidado que tienen con la salud bucal de sus hijos (tabla 6), todos consideran que llevarlos periódicamente al odontólogo es esencial para el cuidado de sus dientes; además, que utilizan crema dental y seda dental en

Tabla 5. Opinión sobre odontólogo y prácticas en salud bucal recibidas en la institución

\begin{tabular}{|c|c|c|}
\hline Variables & Niños $(n=48)$ & Padres $(n=24)$ \\
\hline \multicolumn{3}{|l|}{ ¿Cree que ir al odontólogo es? (Respuestas afirmativas) } \\
\hline Necesario & 37,5 & 62,5 \\
\hline Costoso & 2,1 & 0,0 \\
\hline Aburridor & 2,1 & 4,2 \\
\hline Doloroso & 2,1 & 8,3 \\
\hline Importante & 54,2 & 41,7 \\
\hline Saludable & 12,0 & 50,0 \\
\hline \multicolumn{3}{|l|}{ ¿Ha recibido información sobre el cuidado de los dientes?* } \\
\hline Sí & 91,7 & 79,2 \\
\hline No & 8,3 & 20,8 \\
\hline \multicolumn{3}{|c|}{ ¿Dónde o de quién ha recibido información sobre el cuidado de los dientes?* } \\
\hline Radio y TV & 8,3 & 0,0 \\
\hline Charlas en EPS & 14,6 & 29,2 \\
\hline Charlas en el colegio & 91,7 & 66,7 \\
\hline Odontólogo & 50,0 & 25,0 \\
\hline \multicolumn{3}{|c|}{$\begin{array}{l}\text { ¿Las charlas y actividades que ha realizado la institución sobre } \\
\text { temas de salud bucal han sido de su agrado? }\end{array}$} \\
\hline Sí & 93,8 & 58,3 \\
\hline No & 6,3 & 41,7 \\
\hline
\end{tabular}

Fuente: elaboración propia 
Tabla 6. Prácticas en salud bucal de los padres y adultos significativos con respecto a los niños y las niñas. Fundación las Golondrinas, Medellín

\begin{tabular}{|c|c|}
\hline Variables & Padres $(n=24)$ \\
\hline \multicolumn{2}{|c|}{ ¿Qué hace para el cuidado de boca y dientes de sus hijos? (respuestas afirmativas) } \\
\hline Cepillarles los dientes con crema dental & 87,5 \\
\hline Cepillarles los dientes sin crema dental & 12,5 \\
\hline Alimentarlos con frutas y verduras & 79,2 \\
\hline Llevarlos al odontólogo periódicamente & 100,0 \\
\hline Pasarles la seda dental & 83,3 \\
\hline Darles leche & 66,7 \\
\hline Controlar consumo de dulces & 75,0 \\
\hline \multicolumn{2}{|l|}{ ¿Cree usted que es necesario el uso del flúor para los niños? } \\
\hline Sí & 33,3 \\
\hline No/no sabe & 66,7 \\
\hline \multicolumn{2}{|c|}{ ¿Ha recibido el niño(a) aplicaciones o enjuagues de flúor en el último año? } \\
\hline Sí & 37,5 \\
\hline No & 32,5 \\
\hline \multicolumn{2}{|l|}{ ¿Cuándo fue la última vez que llevó al niño al odontólogo? } \\
\hline 3 meses & 50,0 \\
\hline 6 meses & 29,2 \\
\hline 1 año & 8,3 \\
\hline Más de un año & 12,5 \\
\hline \multicolumn{2}{|l|}{ ¿Cuál fue el motivo de la visita? } \\
\hline Revisión o examen & 58,3 \\
\hline Urgencia & 4,2 \\
\hline Limpieza & 20,8 \\
\hline Operatoria-Endodoncia & 16,7 \\
\hline \multicolumn{2}{|l|}{ Tratamiento de conductos } \\
\hline \multicolumn{2}{|l|}{ ¿Ha notado algún cambio positivo en la salud bucal de su hijo? } \\
\hline Sí & 83,3 \\
\hline No & 16,7 \\
\hline
\end{tabular}

Fuente: elaboración propia

un $88 \%$ y $83 \%$, respectivamente, para complementar el cuidado de los dientes de los niños y niñas. Se encontró también que el $67 \%$ de los adultos encuestados no sabe para qué se utiliza el flúor o si es necesario en el cuidado de los dientes; aun así, el 38\% de los hijos ha recibido topicaciones con flúor en el último año.

Es importante resaltar que se ha generado conciencia en los padres, en cuanto a la frecuencia con que se debe visitar al odontólogo y las razones preventivas por las que se debe hacer, puesto que se observa que $50 \%$ de estos llevó a los escolares hace tres meses al odontólogo, y los motivos más frecuentes fueron revisión o examen en $58 \%$ y limpieza en $21 \%$. Finalmente, las encuestas arrojan que el programa ha tenido un impacto positivo, ya que el $83 \%$ de los padres ha notado un cambio benéfico en la salud bucal de los niños a cargo.

\section{Creencias en salud bucal de los padres y adultos significativos}

Todos los padres y adultos encuestados concuerdan en que ellos son los responsables de enseñarles a los niños a cuidar su boca, y que además deben supervisar el cepillado de los dientes; también concuerdan en que el exceso en el consumo de golosinas aumenta las posibilidades de padecer caries. Para el 96\% de los adultos los niños son más propensos a padecer caries dental, y dormir con el tetero en la boca es perjudicial para su salud bucal. La mayoría de los padres manifiesta que el cuidado de la boca del bebé comienza con la salida del primer diente, y que la alimentación influye en la formación de estos; también indica que la salida de los dientes enferma a los niños. Es importante resaltar que 
Tabla 7. Creencias en salud bucal por padres y adultos significativos. Fundación las Golondrinas, Medellín

\begin{tabular}{|l|c|c|c|}
\hline \multicolumn{1}{|c|}{ Variables } & De acuerdo & En desacuerdo & No sabe \\
\hline En cada embarazo se pierde un diente & 8,3 & 70,8 & 20,8 \\
\hline La primera muela para toda la vida sale antes de los 8 años & 20,8 & 37,5 & 41,7 \\
\hline El cuidado de la boca del bebé comienza con la salida del primer diente & 70,8 & 20,8 & 8,3 \\
\hline Los padres son los responsables de enseñarle al niño(a) a cuidar su boca & 100,0 & 0,0 & 0,0 \\
\hline Los padres deben supervisar el cepillado de los dientes de los hijos(as) pequeños(as) & 100,0 & 0,0 & 0,0 \\
\hline Los niños son más propensos a la caries dental & 95,8 & 0,0 & 4,2 \\
\hline Es dañino o perjudicial para el niño dormir con el tetero en la boca & 95,8 & 4,2 & 0,0 \\
\hline La salida de los dientes enferma a los niños(as) & 83,3 & 12,5 & 4,2 \\
\hline La alimentación influye en la formación de los dientes & 75,0 & 16,7 & 8,3 \\
\hline El exceso en el consumo de golosinas aumenta las posibilidades de padecer caries & 100,0 & 0,0 & 0,0 \\
\hline
\end{tabular}

Fuente: elaboración propia

gran parte de los adultos no está de acuerdo con que en cada embarazo se pierde un diente, y casi la mitad de ellos desconoce la edad en la que erupciona el primer molar permanente (tabla 7 ).

\section{Discusión}

Los resultados de este estudio dan cuenta de la evaluación del componente bucal en el proyecto implementado en la Fundación las Golondrinas en convenio con la Facultad de Odontología de la Universidad de Antioquia. Se puede evidenciar cómo los hallazgos principales señalan una mejoría en cuanto a los conocimientos y prácticas de los escolares que hasta el momento han sido participes del desarrollo del proyecto, haciéndose visible para los adultos que notan un cambio positivo en la salud bucal de los niños. Se observó que el cambio positivo en el IHo es más alto en las mujeres y en los estudiantes mayores de 11 años. En relación con el grado escolar, cabe recalcar que la población de secundaria mostró un cambio levemente mayor que la población de primaria. Finalmente, al observar la población en general, se encontró un cambio positivo en un poco más de la mitad de los niños y las niñas.

Las diferencias respecto al sexo se pueden explicar a partir del reconocimiento que se puede hacer del papel que desempeña la mujer dentro de la sociedad, y de cómo a estas, desde pequeñas, se les imparten ciertos lineamientos de autocuidado que, intencionados o no, ocasionan que la mujer sea más cauta y disciplinada en los asuntos de salud y belleza. Esto se corrobora con otros estudios que demuestran que las mujeres tienen mejores hábitos de higiene bucal cuando se comparan con los hombres [19-22].
Aunque la definición de quién es el odontólogo no concuerde entre niños y adultos, ya que para el $50 \%$ de los primeros es "la persona que enseña cómo cuidarse los dientes" y para el 70\% de los segundos es "la persona encargada de vigilar y controlar que las personas conserven una buena salud oral", en general tienen un concepto acertado del papel que representa el odontólogo en el proceso salud-enfermedad, y el aporte que hace a la conservación de una buena salud bucal.

En la población estudiantil aún no se tienen conceptos claros y definidos acerca de qué es la higiene bucal y su objetivo, mientras que tanto adultos como escolares reconocen términos como la placa bacteriana, la caries dental, su origen, características sobre qué puede conllevar, pero es de aclarar que en la Fundación las Golondrinas se busca motivar a estos escolares por medio de proyectos como el Cepillero, para que reconozcan y hagan un uso adecuado de los implementos de higiene bucal como el cepillo, la crema y la seda dental, lo que ha permitido en parte el cambio positivo en los indicadores del iHos.

De otra parte, los padres piensan que visitar al odontólogo es costoso, aburridor y doloroso, pero reconocen la importancia y necesidad de esas acciones para conservar una buena salud bucal, y están de acuerdo en llevar periódicamente a sus hijos al odontólogo, mientras que la mayoría de escolares no muestra una postura definitiva, ya que piensa que la consulta odontológica es necesaria pero no saludable, tampoco la relacionan con altos costos, ni como una visita aburridora y dolorosa. Se podría decir entonces que es preciso reforzar, tanto en los niños como en los padres, el concepto de salud bucal y su importancia dentro la salud general, y a partir de ello puedan ambos coincidir en algún momento en cuanto a que visitar al odontólogo es una necesidad. 
Otro hallazgo importante encontrado en esta investigación radica en que no se observaron cambios significativos en algunas variables relacionadas con el COP-D de estos escolares, pero esto finalmente se explica a partir de la intención de la Fundación las Golondrinas de generar espacios de medicina general, odontología, nutrición, entre otros servicios, los cuales en ningún momento buscan solucionar los problemas de accesibilidad a los servicios de salud de la población vinculada a esta institución, sino más bien propiciar espacios en los que la promoción de la salud y la prevención de la enfermedad sean los ejes fundamentales para empoderar a la población en el cuidado de su salud.

Cabe también destacar el aporte que esta fundación hace a través del convenio con la Facultad de Odontología, lo cual ha permitido la transmisión de información y conocimientos tanto a los estudiantes como a sus familias en el cuidado de la salud bucal. En este punto es preciso mencionar el escaso aporte que hacen los medios de comunicación y las Empresas Promotoras de Salud (EPS) con respecto a la salud bucal, en opinión de los escolares y padres de familia encuestados.

Según la Organización Mundial de la Salud (OMs) [23], la meta establecida a nivel mundial para el indicador de COP-D en el 2000 debería ser menor de 3,0, y para Colombia, según el Estudio Nacional de Salud Bucal III (Ensab III) [24], el indicador debe estar por debajo de 2,3. Teniendo en cuenta estos datos, y al compararlos con los resultados de este estudio, se observa que los índices de la población estudiada están por debajo de estos promedios. En cuanto a los índices de placa bacteriana, el Ensab III indica que para las edades de 5 a 14 años el promedio debe ser 1,45. Al confrontar esta información con el estudio realizado en la fundación, se observaron leves diferencias, mientras que en los estudiantes de 7 a 10 años se halló un índice de 1,49; los estudiantes de 11 años o mayores presentaron una leve disminución, su índice fue 1,28 , y según la escala de valoración para el IHO la higiene bucal de esta población se encuentra entre buena y regular [14,24]. También fue posible comparar los resultados respecto a conocimientos y prácticas de salud bucal con los resultados del Ensab III, ya que gran parte de las preguntas que se realizaron estuvieron basadas en este estudio, y se evidencia en términos generales que existe claridad sobre los conceptos básicos de salud bucal y las actividades que se deben realizar para mantener una higiene bucal adecuada [24].

En un estudio realizado por González y colaboradores en un colegio de la ciudad de Medellín [25] también se evidencia la disminución en los índices de placa bacteriana y el CoP-D, además de un cambio positivo en relación con la percepción y los conocimientos sobre salud bucal tras implementar un proyecto de educación para la salud; lo cual concuerda con la literatura internacional [7-13]. Cabe resaltar el cambio en la percepción de los padres sobre la necesidad de que los niños tengan visitas periódicas con el odontólogo y sus motivos de consulta, ya que la mayoría manifestó que la última visita fue "hace tres meses", y que el motivo de consulta fue "revisión y limpieza". Este hecho se evidencia también en un estudio realizado por González y colaboradores en Cartagena [26], donde el 46,2\% de los padres manifestó que este es el principal motivo de consulta, dejando el dolor en un segundo plano, puesto que sólo el 19,6\% expresó que ese fuera su motivo de consulta. También en un estudio realizado por Saldarriaga y colaboradores con madres gestantes vinculadas a un programa de control prenatal en Medellín, el $48,6 \%$ de estas señala que el principal motivo de consulta es control y prevención [27].

Es de suma importancia mencionar las principales fortalezas y limitaciones que tuvo el estudio durante su desarrollo. Esta investigación ha tenido gran apoyo y acompañamiento por parte de la Facultad de Odontología de la Universidad de Antioquia, que permitió la participación activa de los investigadores en diferentes actividades de reconocimiento en la Fundación las Golondrinas, siendo esto de gran ayuda para el logro de los resultados obtenidos, además de la disposición de las áreas de trabajo que fueron facilitadas por la Fundación para la ejecución de las diferentes acciones. Igualmente, se trató de evaluar diferentes variables, no sólo clínicas sino de conocimientos y prácticas, lo que permitió la integración de varios determinantes de salud. También es preciso tener en cuenta las debilidades y hacer una intervención de estas para que en próximas investigaciones se puedan obtener mejores resultados: hubo baja participación de los padres o adultos significativos en la investigación, lo que dificultó la integración de los conocimientos y la ejecución de algunas actividades; por otro lado, la falta de continuidad de los profesores en la institución obstaculizó el acompañamiento de los estudiantes durante todo el proceso; por último, se debe tener presente la deserción estudiantil que, además de afectar la muestra del estudio, podría infra o supraestimar sus resultados.

Esta investigación es posible complementarla con otros estudios que permitan caracterizar mejor la comunidad y evaluar otras variables como los factores de riesgo que contribuyen con el incremento de la preva- 
lencia y experiencia de caries dental. Debido a la poca participación de los padres, sería ideal indagar y entender más a fondo los conocimientos, actitudes y prácticas de estos en cuanto a salud bucal; también sería importante conocer las razones por las cuales la gente realmente no busca atención clínica ni asiste a los centros de salud. Dado que la fundación brinda otros servicios a la comunidad, como por ejemplo: medicina, nutrición y psicología, se podría realizar un estudio multidisciplinario que integre todas las áreas de la salud para brindar una mejor atención a la población.

\section{Conclusión}

Gracias a que el desarrollo del proyecto se ha centrado en la promoción de la salud y en la prevención de la enfermedad, se evidencia una leve disminución en la prevalencia de caries dental, pero es importante que la fundación ejecute un programa con el cual se pueda llevar un mejor control y seguimiento de los estudiantes y sus condiciones de salud bucal. En primer lugar, es necesario interpretar los resultados obtenidos con el proyecto escuela saludable, implementado e integrado al Proyecto Educativo Institucional (PEI), mirado desde una perspectiva de educación más que de curación, y resaltando que este tipo de proyectos, que buscan cambiar modos de vida en una comunidad, requieren largo tiempo y trabajo conjunto como el que se ha venido desarrollando. Puede decirse, entonces, que se han logrado en cierta medida cambios muy positivos que nos conducen a esos primeros pasos para la transformación que se desea en esta comunidad. Hay que resaltar y reconfirmar que no es posible intervenir los problemas bucales solamente desde una óptica individual, que es necesario un conjunto de estrategias conformadas por aspectos sociales, políticos, educativos y económicos para lograr un verdadero cambio social significativo [28]. Para terminar, evidenciamos la necesidad de complementar la parte educativa que se está llevando a cabo, con la parte clínica curativa, para lograr integrar adecuadamente los conceptos de promoción de la salud, prevención de la enfermedad y curación.

\section{Agradecimientos}

Al personal de la Fundación las Golondrinas, por facilitar las instalaciones y la logística necesaria para el desarrollo de la investigación en cada una de sus fases, y a los escolares y padres y adultos significativos, quienes participaron desinteresadamente brindando la información necesaria para el desarrollo del trabajo de campo.

\section{Referencias}

[1] Organización Mundial de la Salud. Ministerio de Salud y Bienestar Social de Canadá. Asociación Canadiense de Salud Pública. Carta de Ottawa para la Promoción de la Salud. Ottawa: Organización Mundial de la Salud; 1986.

[2] WHo Expert Committee on Comprehensive School Health Education and Promotion. Promoting health through schools. [Internet]. Geneva: World Health Organization; 1997. [Consultado: 30 de marzo de 2012]. Disponible en: http://whqlibdoc.who.int/trs/WHO_ TRS_870.pdf

[3] Kwan SY, Petersen PE, Pine CM, Borutta A. Healthpromoting schools: an opportunity for oral health promotion. Bull World Health Organ. 2005; 83(9): 677-85.

[4] Martínez-Herrera E, Agudelo-Suárez A, González-Ariza S. El mercadeo social: una disciplina que fortalece el fomento de la salud bucal en Colombia. Rev ces Odont. 2009; 22(2): 77-84.

[5] Fundación las Golondrinas. [Internet]. Medellín: La Fundación; 2011. [Consultado: 30 de marzo de 2012]. Disponible en: http://www.fundacionlasgolondrinas.org/ main/

[6] Universidad de Antioquia. Facultad de Odontología. Convenio pasantías estudiantiles No 3233/002/2006 celebrado entre la Universidad de Antioquia (Facultad de Odontología) y la Fundación las Golondrinas NIT. 800009090-6. Medellín: Facultad de Odontología; 2006.

[7] Álvarez CJ, Navas R, Rojas T. Componente educativorecreativo-asociativo en estrategias promotoras de salud bucal en preescolares. Rev Cubana Estomatol [Internet] 2006; 43(2) [Consultado: 30 de marzo de 2013]. Disponible en: http://scielo.sld.cu/scielo.php?script=sci_ar ttext\&pid=S0034-75072006000200005

[8] Murrieta Pruneda JF, Juárez López LA, Linares Vieyra C, Zurita Murillo V. Prevalencia de gingivitis en un grupo de escolares y su relación con el grado de higiene oral y el nivel de conocimientos sobre salud bucal demostrado por sus madres. México. Bol Med Hosp Infant Mex. 2004; 61(1): 44-54.

[9] Limonta Vidal ER, Araújo Heredia T. Intervención educativa para modificar conocimientos sobre salud bucal en escolares de tercer grado. Medisan 2000; 4(3): 9-15.

[10] Caldés Ruisánchez S, Cea Sánchez N, Crespo Aliseda P, Díez Nicolás V, Espino García A, Galán Arévalo S 
et al. ¿Una intervención educativa en niños de doce años de Madrid modifica sus conocimientos y hábitos de higiene buco-dental? Av Odontoestomatol 2005; 21-3: 149-57.

[11] Bernabé-Ortiz E, Sánchez-Borjas PC, Delgado-Angulo EK. Efectividad de una intervención comunitaria en salud oral: resultados después de 18 meses. Rev Med Hered. 2006; 17(3): 170-6.

[12] Medina CE, Segovia A, Estrella R, Maupomé G, Ávila L, Pérez R. Asociación del nivel socioeconómico con la higiene bucal en preescolares bajo el programa de odontología preventiva del IMss en Campeche. México. Gac Med Mex. 2006; 142(5): 363-8.

[13] Tubert-Jeannin S, Leger S, Manevy R. Addressing children's oral health inequalities: Caries experience before and after the implementation of an oral health promotion program. Acta Odontol Scand. 2012; 70(3): 255-64.

[14] Green JC. Vermillion JR. The simplified oral hygiene index. J Am Dent Assoc. 1964; 68: 7-13.

[15] Klein H, Palmer CE, Knutson JW. Studies on Dental Caries, I, Dental status and dental needs of elementary school. Public Health Report. 1938; 53: 751-65.

[16] Gruebbel AO. A measurement of dental caries prevalence and treatment service for deciduous teeth. J Dent Res. 1944; 23: 163-8.

[17] World Medical Association General Assembly. World Medical Association Declaration of Helsinki: ethical principles for medical research involving human subjects. J Int Bioethique. 2004; 15(1): 124-9.

[18] República de Colombia. Ministerio de Salud y Protección Social. Resolución nº 008430 de 1993 (4 de octubre de 1993) por la cual se establecen las normas científicas, técnicas y administrativas para la investigación en salud. Bogotá: El Ministerio; 1993.

[19] Mazarro Bodega G. Análisis de las diferencias de género en el cuidado bucodental. Gaceta Dental. 2012; 241: 114-25.
[20] Al-Ansari JM, Honkala S. Gender differences in oral health knowledge and behavior of the Health Science College students in Kuwait. J Allied Health. 2007; 36(1): 41-6.

[21] Kakuhino F, Yushinori T, Yoshinobu M. Gender differences in oral health behavior and general health habits in an adult population. Bull Tokyo Dent Coll. 1999; 40(4): 187-93.

[22] Sakalauskiene Z, Vehkalahti MM, Murtonaa H, Maciulskiene V. Factors related to gender differences in toothbrushing among Lithuanian middleaged university employees. Medicina (kaunas). 2011; 47(3): 180-6.

[23] Organización Mundial de la Salud. Federación Dental Internacional. Metas de salud bucal para el año 2000. Ginebra: OMS-FDI; 1979.

[24] Colombia. Ministerio de Salud. III Estudio Nacional de Salud Bucal Ensab III, 1998. Bogotá: Ministerio de Salud; 1998.

[25] González S, Álvarez L, Amaya A, Atuesta C, Betancur G, Maya D, Múnera T. Promoción y prevención en salud bucal en docentes y escolares de una escuela de Medellín. Revista Colombiana de Investigación en Odontología. 2009; 1(1) supl: 46-57.

[26] González-Martínez F, Sierra-Barrios CC, Morales-Salinas LE. Conocimientos, actitudes y prácticas en salud bucal de padres y cuidadores en hogares infantiles, Colombia. Salud Publica Mex. 2011; 53(3): 247-57.

[27] Saldarriaga O, Sánchez M, Avendaño L. Conocimientos y prácticas en salud bucal de las gestantes vinculadas al programa de control prenatal. Medellín 2003. Rev CES Odontol. 2004; 17(2): 34-44.

[28] Agudelo A, Martínez E. La salud bucal colectiva y el contexto colombiano: un análisis crítico. Rev Gerenc Polit Salud. 2009; 8(16): 91-105. 